\title{
PRECESSION OF THE $\epsilon$ RING OF URANUS
}

\author{
Peter Goldreich \\ Division of Geological and Planetary Sciences, California Institute of Technology, Pasadena, California 91125
}

\author{
Scott Tremaine
}

Institute for Advanced Study, Princeton, New Jersey 08540

Received 4 June 1979

\begin{abstract}
The boundaries of the $\epsilon$ ring can be fit by aligned Keplerian ellipses. Differential precession due to the quadrupole moment of Uranus tends to destroy the apse alignment. We propose that apse alignment is maintained by the self-gravity of the ring. The required ring mass $\sim 5 \times 10^{18} \mathrm{~g}$ which corresponds to a surface density at quadrature $\sim 25 \mathrm{~g} \mathrm{~cm}^{-2}$.
\end{abstract}

\section{INTRODUCTION}

One of the remarkable features of the Uranus ring system is that several of the rings are not circular. The outer and inner boundaries of the $\varepsilon$ ring can be fit by aligned Keplerian ellipses with eccentricities $\mathrm{e}_{0} \pm \delta \mathrm{e} / 2$ and semimajor axes $a_{0} \pm \delta a / 2$, where (Nicholson et al. 1978)

$$
\begin{gathered}
e_{0}=7.80 \times 10^{-3}, \\
\delta e=7.2 \times 10^{-4}, \\
a_{0}=51284 \mathrm{~km}, \\
\delta a=60 \mathrm{~km} .
\end{gathered}
$$

The $\alpha$ ring is also elliptical, but it is narrower and has not been resolved. Its mean eccentricity is $6.3 \times 10^{-4}$ and its mean semimajor axis is $44,839 \mathrm{~km}$.

The origin of the ring eccentricities is not yet understood. In spite of this, it is possible to investigate how an elliptical ring maintains apse alignment.

The apsidal line of a low-eccentricity orbit around an oblate planet precesses at a rate

$$
\frac{d \tilde{\omega}}{d t}=\frac{3}{2} J_{2} R^{2}(G M)^{1 / 2} a^{-7 / 2} .
$$

Here $a$ is the semimajor axis, and $M, R$, and $J_{2}$ are the mass, radius, and dynamical oblateness of the planet. For Uranus $J_{2} R^{2}=3.43 \times 10^{-3}(26,200 \mathrm{~km})^{2}$ (Nicholson et al. 1978). Equation (2) shows that in the absence of extra forces the inner edge of the $\varepsilon$ ring would precess one revolution relative to the outer edge in 175 years. Thus, the observed alignment must be explained.

In Sec. II we consider the possibility that the particle apsides are locked together by the ring's self-gravity. Other possible explanations are discussed in Secs. III and $\mathrm{IV}$, and Sec. V contains a discussion of our results.

\section{THE SELF-GRAVITY OF THE RING}

We represent the ring as a collection of elliptical wires, whose linear density at any point is inversely proportional to the local particle velocity. For a wire of total mass $m$, eccentricity $e$ and semimajor axis $a$, the linear density at true anomaly $f$ is

$$
\rho \cong \frac{m}{2 \pi a}(1-e \cos f)+O\left(e^{2}\right) .
$$

We calculate the forces exerted by this wire on a sample ring particle with semimajor axis $a_{p}=a-\Delta a$ and eccentricity $e_{p}=e-\Delta e$. We assume that the apsides of the particle and the wire are aligned since we are only interested in the maintenance of this alignment. Also, since the observed rings are narrow we assume $|\Delta a / a|$ $\ll 1$. by

The geometry is shown in Fig. 1. The angle $\phi$ is given

$$
\phi \cong e \sin f+O\left(e^{2}\right) .
$$

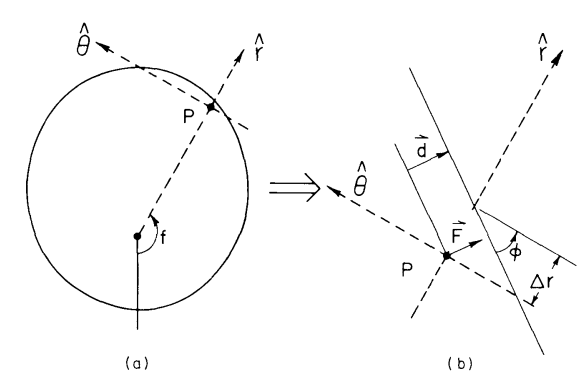

FIG. 1. The geometry of the particle-wire interaction. Figure 1(b) is a magnified view of the region of Fig. 1(a) near the particle $p$. In Fig. 1(a) the wire eccentricity $e$ and the relative distance between the particle and the wire are much larger than in the real ring system. The wire eccentricity has been exaggerated even more in Fig. 1(b) for clarity. 
The perpendicular from the particle to the wire is d, where

$$
\begin{gathered}
d=|\mathbf{d}|=\Delta r \cos \phi \\
=\left|\left(a-a_{p}\right)+\left(a_{p} e_{p}-a e\right) \cos f\right|+O\left(e^{2}, e_{p}^{2}\right) \\
=|\Delta a|(1-q \cos f)+O\left(e^{2}, e_{p}^{2}\right),
\end{gathered}
$$

where $q+(a \Delta e+e \Delta a-\Delta a \Delta e) / \Delta a$. We assume that the orbits do not cross, i.e., $|q|<1$.

The wire is approximately straight in the neighborhood of the particle. Thus, the gravitational acceleration due to the wire is $\mathbf{F}=2 G \rho \mathbf{d} / d^{2}=S \hat{r}+T \hat{\theta}$, where

$$
\begin{gathered}
S \cong 2 G \rho \operatorname{sgn}(\Delta a) / d+O\left(e^{2}\right), \\
T=-2 G \rho \operatorname{sgn}(\Delta a) e \sin f / d+O\left(e^{2}\right) .
\end{gathered}
$$

The apse precession rate due to these forces is (Brouwer and Clemence 1961)

$$
\begin{aligned}
\frac{d \tilde{\omega}_{p}}{d t}=\frac{1}{n_{p} a_{p} e_{p}}[ & -S \cos f \\
& \left.+T \sin f\left(2-e_{p} \cos f\right)\right]+O\left(e_{p}\right),
\end{aligned}
$$

where $n_{p}=\left(G M / a_{p}^{3}\right)^{1 / 2}$ is the mean motion. Combining Eqs. (3), (5), (6), and (7) yields

$$
\begin{aligned}
\frac{d \tilde{\omega}_{p}}{d t} & =-\frac{1}{\pi} \frac{m}{M} \frac{n_{0} a_{0}}{e_{p} \Delta a} \\
& \times \frac{\left(\cos f-e \cos ^{2} f+2 e \sin ^{2} f\right)}{1-q \cos f}+O\left(e, e_{p}, \frac{e^{2}}{e_{p}}\right) .
\end{aligned}
$$

In Eq. (8) we have replaced $n_{p}, a, a_{p}$ by $n_{0}$ and $a_{0}$ [cf. Eq. (1)] since $|\Delta a| / a \ll 1$. Since $d f / d t=n_{p}\left(1+2 e_{p} \cos f\right)$ $+O\left(e_{p}^{2}\right)$, the average precession rate is

$$
\begin{aligned}
&\left\langle\frac{d \tilde{\omega}_{p}}{d t}\right\rangle=-\frac{1}{\pi^{2}}\left(\frac{m}{M}\right) \frac{n_{0} a_{0}}{e_{p} \Delta a} \int_{0}^{\pi} \\
& \times d f \frac{\cos f+2 e-\left(2 e_{p}+3 e\right) \cos ^{2} f}{1-q \cos f} \\
&+O\left(e, e_{p}, \frac{e^{2}}{e_{p}}\right) .
\end{aligned}
$$

The terms which are $O\left(e, e_{p}, e^{2} / e_{p}\right)$ may be dropped. The result of this integration is

$$
\begin{aligned}
\left\langle\frac{d \tilde{\omega}_{p}}{d t}\right\rangle & =-\frac{1}{\pi}\left(\frac{m}{M}\right) \frac{n_{0} a_{0}}{e \Delta a \cos 2 \psi} \\
& \times\left[\frac{1}{2} e-e_{p}+\tan \psi-\left(e_{p}+\frac{3}{2} e\right) \tan ^{2} \psi\right],
\end{aligned}
$$

where $q=\sin 2 \psi$. A version of Eq. (10) valid in the limit $|\Delta e| \ll e$ is given as Eq. (13) of Goldreich and Tremaine (1979). Note that as $\Delta e$ approaches zero the right-hand side of Eq. (10) is $O\left(e^{2}\right)$. Thus, Eq. (10) is not valid when $\Delta e$ is very small $\left(\Delta e \lesssim e^{2} \Delta a / a\right)$, since terms which we have neglected dominate the precession rate. This limitation is not important.in applications to the $\varepsilon$ ring.

We shall use the complete Eq. (10) in Sec. III. However, in this section a simpler form is sufficient. For the $\varepsilon$ ring $a \delta e / e_{0} \delta a=78.9$ [cf. Eq. (1)]. Consequently, we assume $a \Delta e / e \Delta a \gg 1$ for pairs of subrings in the $\varepsilon$ ring. Thus, $q \cong a \Delta e / \Delta a$ and the term $\tan \psi$ dominates the square bracket in Eq. (10). Finally, we replace the multiplicative factor $1 / e_{p}$ in Eq. (10) by $1 / e_{0}$ since $|\Delta e|$ $\ll e$, and obtain

$$
\left\langle\frac{d \tilde{\omega}_{p}}{d t}\right\rangle=-\frac{1}{\pi} \frac{m}{M} \frac{n_{0} a_{0} \tan \psi}{e_{0} \Delta a \cos 2 \psi} .
$$

Next we sum the contributions to the precession from all parts of the ring. Let the semimajor axes of the inner and outer boundaries of the ring be $a_{\text {in }}$ and $a_{\text {out }}=a_{\text {in }}+$ $\delta a$. Divide the region $a_{\text {in }}$ to $a_{\text {out }}$ into $N$ equal intervals of width $\delta a / N$, each of which contains a wire of mass $m_{j}$, semimajor axis $a_{j}=a_{\mathrm{in}}+(\mathrm{j}-1 / 2) \delta a / N$, and eccentricity $e_{j}, j=1, \ldots, N$. We write $m_{j}=h_{j} m_{r}$, where $m_{r}$ is the total ring mass and

$$
\sum_{j=1}^{N} h_{j}=1
$$

The precession rate of wire $j$ due to all other wires is

$$
\frac{d \tilde{\omega}_{j}}{d t}=-\frac{n_{0} a_{0} N}{\pi e_{0} \delta a} \frac{m_{r}}{M} \sum_{k \neq j} \frac{h_{k}}{k-j} \frac{\tan \psi_{j k}}{\cos 2 \psi_{j k}},
$$

where

$$
\sin 2 \psi_{j k}=\frac{N a_{0}}{\delta a}\left(\frac{e_{k}-e_{j}}{k-j}\right) .
$$

The precession rate due to the quadrupole moment of Uranus is

$$
\left(\frac{d \tilde{\omega}_{j}}{d t}\right)_{Q}=\text { const }-\frac{21}{4} J_{2}\left(\frac{R}{a_{0}}\right)^{2} \frac{n_{0}}{N} j \frac{\delta a}{a_{0}} .
$$

The condition that the ring precess uniformly is

$$
j \frac{C}{N}+N \sum_{k \neq j} \frac{h_{k}}{k-j} \frac{\tan \psi_{j k}}{\cos 2 \psi_{j k}}=D, \quad j=1, \ldots, N,
$$

where $D$ is a constant and $C=(21 \pi / 4) e_{0} J_{2}\left(M / m_{r}\right)$ $\times\left(R / a_{0}\right)^{2}\left(\delta a / a_{0}\right)^{2}$. The values of $e_{1}$ and $e_{\mathrm{N}}$ are the eccentricities of the ring boundaries. Thus, there are $N$ equations for the $N$ unknowns $C, D, e_{2}, \ldots, e_{N-1}$. The ring mass is determined from $C$.

We have solved these equations for four mass distributions $\left\{h_{k}\right\}$ : (a) flat distribution $h_{k}=1 / N$, (b) parabolic distribution $h_{k}=A-B\left(k-k_{0}\right)^{2}$, where the constants $A, B$, and $k_{0}$ are chosen so that the ring is symmetrical with central surface density equal to twice the edge surface density at quadrature; (c) the same distribution as in case (b) but with central surface density equal to half the edge surface density; (d) a profile chosen to be similar to the profile observed by Nicholson et al. (1978). The ring mass in cases (a)-(d) is $4.63 \times 10^{18} \mathrm{~g}, 4.20 \times$ $10^{18} \mathrm{~g}, 5.28 \times 10^{18} \mathrm{~g}$ and $4.83 \times 10^{18} \mathrm{~g}$, respectively. These results were obtained for $N=80$ wires and should be accurate to about $1 \%$. The corresponding surface density distributions at pericenter, quadrature and apocenter are shown in Fig. 2. 
surface

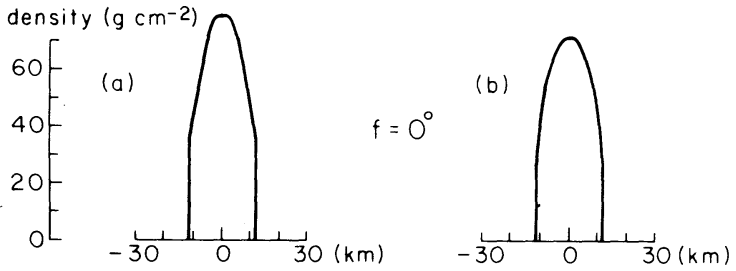

205

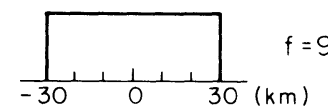

${ }^{20}[$
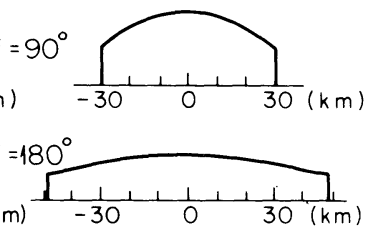

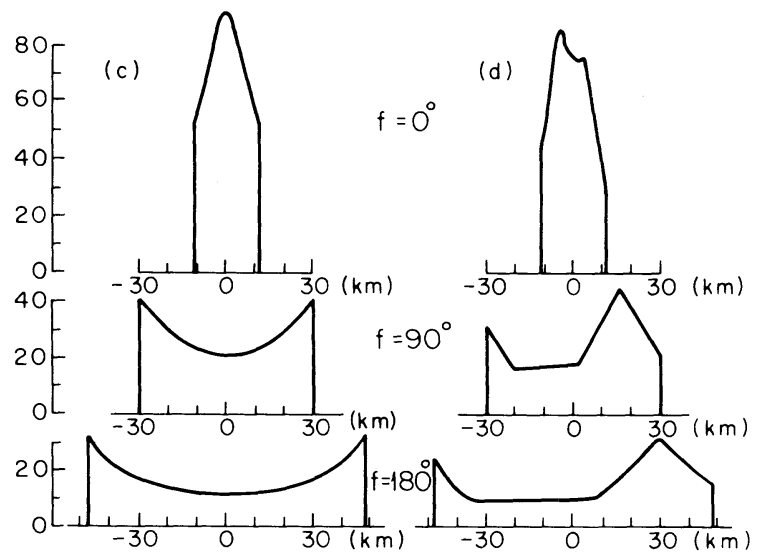

FIG. 2. Surface density at pericenter $\left(f=0^{\circ}\right)$, quadrature $\left(f=90^{\circ}\right)$, and apocenter $\left(f=180^{\circ}\right)$ for rings which precess uniformly under self-gravity. The abscissa is radial distance from the ring center.

It is worth noting that our model requires $e_{N}-e_{1}=$ $\delta e>0$ as observed. The solution of eqs. (14) with the sign of $\delta e$ reversed does not yield a positive definite ring mass in any of the four cases. There is a simple physical explanation of why $\delta e$ must be positive. Consider a particle at the inner boundary of the ring. The ring's gravity must retard its apse precession rate. The gravitational force from the ring is approximately radially outward. Such a force must be stronger at pericenter than at apocenter in order to retard the precession. Thus, the ring must be narrower at pericenter than at apocenter which is equivalent to $\delta e>0$. Consideration of particles at the outer boundary leads to the same requirement.

\section{PRECESSION DUE TO A SATELlite}

Goldreich and Tremaine (1978) have argued that the Uranian rings are supported against collisional diffusion and radiation drag by small satellites orbiting between the rings. In this section we investigate whether such satellites could force uniform precession in the $\varepsilon$ ring.

\section{a) Satellite and Ring Precess Together}

The simplest possibility is that the satellite and ring particle apsides are aligned and precess at the same rate. (An example of this kind of behavior is given by the satellites Titan and Rhea.) Equations (2) and (10) determine the precession rate of a ring particle relative to the satellite:

$$
\begin{aligned}
\left(\frac{d \tilde{\omega}_{p}}{d t}-\frac{d \tilde{\omega}_{s}}{d t}\right) & =\frac{21}{4} J_{2}\left(\frac{R}{a_{s}}\right)^{2} n_{s} \frac{\Delta a}{a_{s}} \\
& -\frac{1}{\pi} \frac{m_{s}}{M} \frac{n_{s} a_{s}}{e_{p} \Delta a \cos 2 \psi}\left[\frac{1}{2} e_{s}-e_{p}+\tan \psi\right],
\end{aligned}
$$

where $\sin 2 \psi=a_{s} \Delta e / \Delta a+e_{p}$. Here the subscripts $s$ and $p$ refer to the satellite and the ring particle, $\Delta a=a_{s}-$ $a_{p}$ and $\Delta e=e_{s}-e_{p}$. The term $\left(e_{p}+3 / 2 e\right) \tan ^{2} \psi$ in Eq. (10) has been dropped because it is negligible compared with $\tan \psi$. We have neglected all forces on the ring particle except the attraction from the satellite and the oblate planet, and we have neglected the effect of the ring on the satellite orbit.

The right-hand side of Eq. (15) must vanish for all ring particles, including those on the ring boundaries. Since we know $e_{p}$ and $a_{p}$ at the boundaries we obtain two constraints on the unknowns $m_{s}, a_{s}$, and $e_{s}$. These constraints are plotted in Fig. 3. We also include the constraint that no part of the satellite can intersect the ring (we determine the satellite radius using a density $\rho_{s}=$ $\left.1 \mathrm{~g} \mathrm{~cm}^{-3}\right)$.

\section{b) Satellite and Ring Precess Independently}

If the satellite and ring precess independently, the eccentricity of the satellite orbit plays only a minor role in determining the precession rate of a ring particle. Hence we set $e_{s}=0$ for simplicity. By analogy with Eq. (15) the relative precession rate of the inner and outer ring boundaries is

$$
\begin{aligned}
\frac{d \tilde{\omega}_{\text {out }}}{d t}-\frac{d \tilde{\omega}_{\text {in }}}{d t}= & -\frac{21}{4} J_{2}\left(\frac{R}{a_{s}}\right)^{2} n_{s} \frac{\delta a}{a_{s}}-\frac{1}{\pi} \frac{m_{s}}{M} n_{s} a_{s} \\
\times\left(\frac{\tan \psi_{\text {out }}-e_{\text {out }}}{e_{\text {out }}\left(a_{s}-a_{\text {out }}\right) \cos 2 \psi_{\text {out }}}\right. & \left.-\frac{\tan \psi_{\text {in }}-e_{\text {in }}}{e_{\text {in }}\left(a_{s}-a_{\text {in }}\right) \cos 2 \psi_{\text {in }}}\right)
\end{aligned}
$$

where $\sin 2 \psi_{i}=-a_{s} e_{i} /\left(a_{s}-a_{i}\right)+e_{i}, i=$ in, out. The condition that the relative precession rate vanish determines the satellite mass as a function of $a_{s}$ (cf. Fig. 3).

\section{INTERPARTICLE COLLISIONS}

\section{a) "Thermal" Pressure}

Interparticle collisions create pressure gradients corresponding to a force per unit mass $\nabla \mathrm{p} / \rho \sim\left\langle v^{2}\right\rangle / l$, where $\left\langle v^{2}\right\rangle$ is the mean-square velocity dispersion and 


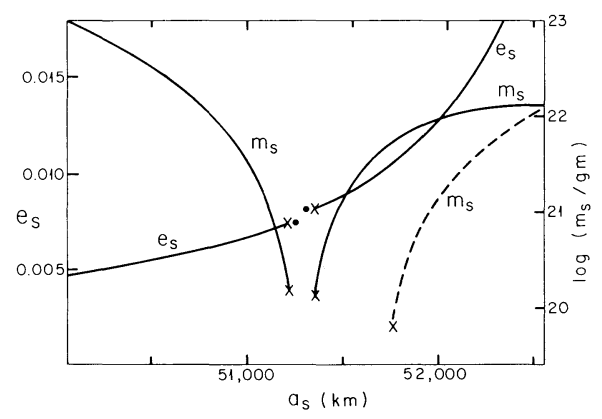

FIG. 3. The parameters of a satellite which would force uniform precession in the $\varepsilon$ ring. The quantities $m_{s}, e_{s}$, and $a_{s}$ are the satellite mass, eccentricity, and semimajor axis. Solid lines are for an apsealigned satellite and dashed lines for a circular satellite $\left(e_{s}=0\right)$. Dots mark the eccentricities and semimajor axes of the ring boundaries. Crosses mark the point where a spherical satellite of density $\rho_{s}=1$ $\mathrm{g} \mathrm{cm}^{-3}$ would collide with the ring.

$l$ is a characteristic dimension (we take $l \sim 60 \mathrm{~km} \sim \varepsilon$ ring width). Replacing the radial force $S$ with $\left\langle v^{2}\right\rangle / l$ in Eq. (7) we find that pressure can induce variations in the precession rate across the ring of order $\Delta\left(d \tilde{\omega}_{p} / d t\right) \sim$ $\left\langle v^{2}\right\rangle /($ lnae $)$. The differential precession across the ring due to the quadrupole moment of Uranus is $\Delta\left(d \tilde{\omega}_{p} / d t\right)$ $\sim-21 / 4 J_{2}(R / a)^{2} n l / a$ [cf. Eq. (2)]. The requirement that the two variations have the same order of magnitude is $\left\langle v^{2}\right\rangle^{1 / 2} \sim 10 \mathrm{~cm} \mathrm{~s}^{-1}$. This dispersion corresponds to a ring thickness $\left\langle z^{2}\right\rangle^{1 / 2} \sim 0.5 \mathrm{~km}$. It is hard to imagine that the ring is this thick; it is more reasonable that the ring is nearly a monolayer so that the thickness is comparable to the particle size (Goldreich and Tremaine 1978).

\section{b) "Shocks"}

A more interesting possibility is that interparticle collisions may have effects which cannot be modelled by smooth pressure gradients. For example, there may be a discontinuity analogous to a shock; or there may be a region where the particles are so tightly packed that they behave like an incompressible fluid.

The precession rate induced by an impulse per unit mass of strength $\Delta I$ per orbit is $d \tilde{\omega} / d t \sim \Delta I /($ ae) [cf. Eq. (7)]. The condition that this precession is of the same order as the differential precession due to the quadrupole moment of Uranus is $\Delta I \sim 0.05 \mathrm{~cm} \mathrm{~s}^{-1}$. It seems possible that the ring may contain shocks which give the ring particles an impulse of this order of magnitude every orbit. However, the physics of these processes is very complex and we have not investigated them in detail.

\section{DISCUSSION}

We have discussed four possible mechanisms for maintaining uniform precession in the $\varepsilon$ ring. We favor self-gravity, since the other possibilities suffer one or more difficulties.

The idea that the ring particle apsides are locked to the apse of a nearby satellite has the attractive feature that the ring eccentricity could be maintained against dissipation. However, the required satellite mass is very much greater than that implied by our confinement theory (Goldreich and Tremaine 1979). Our theory predicts that the ring would be rapidly repelled by the satellite.

If the ring apsides are not locked to the satellite apse, the required satellite mass is smaller, at least for small satellite-ring separations. This weakens but does not remove the conflict with our confinement theory.

We have shown that smooth pressure gradients cannot produce uniform precession without an unreasonably large ring thickness. However, the possibility that shocklike phenomena can force uniform precession is difficult to analyze and thus cannot be dismissed.

If the uniform precession is due to self-gravity, the ring mass is $\sim 5 \times 10^{18} \mathrm{~g}$ and its mean surface density at quadrature is $\sim 25 \mathrm{~g} \mathrm{~cm}^{-2}$. This model predicts a strong variation in surface density profile as a function of true anomaly (cf. Fig. 2) as a consequence of the nonuniform eccentricity gradient across the ring. This prediction can be tested by future occultation observations of the ring profile.

This research was supported by NASA Grant NGL-002-003 and NSF Grants AST78-21453 and PHY77-20612.

\section{REFERENCES}

Brouwer, D. and Clemence, G. (1961). Methods of Celestial Mechanics (Academic, New York).

Goldreich, P., and Tremaine, S. (1978). Icarus 34, 227.
Goldreich, P., and Tremaine, S. (1979). Nature 277, 97.

Nicholson, P. D., Persson, S. E., Matthews, K., Goldreich, P., and Neugebauer, G. (1978). Astron J. 83, 1240. 